\title{
The Impact of the US Dollar and the Euro on Currencies in Europe and Asia
}

\author{
Prof. Dr. Malgorzata Doman (Poznan University of Economics, Poland) \\ Prof. Dr. Ryszard Doman (Adam Mickiewicz University, Poland)
}

\begin{abstract}
In this paper, we analyze dependencies between the currencies of chosen emerging countries and the major (global) currencies - the euro and the American dollar. The idea is taken from a paper by Eun and Lai proposing a method to verify an opinion that currencies systematically co-move and the pattern of co-movement is significantly driven by the relative influence of the two global currencies. The observation by Eun and Lai is that in the case when a minor currency XYZ is driven by the US dollar, the exchange rates XYZ/EUR and USD/EUR co-move very closely. In the opposite case, i.e. when the $\mathrm{XYZ}$ is influenced by the euro, the exchange rates $\mathrm{XYZ/USD}$ and EUR/USD show strong interdependence. In our approach, the dynamics of dependencies is modeled by means of 3-regime Markov regime switching copula models, and the considered measures of the strength of the linkages are dynamic Spearman's rho and tail dependence coefficients. Applying the Markov regime switching copula models allows us to capture temporal changes in the impact of the global currencies on the analyzed minor ones. Our results show that the euro area of influence is widening, and that during the considered period some of the analyzed currencies are releasing from the US dollar impact.
\end{abstract}

\section{Introduction}

In the paper, we analyze dependencies between the currencies of chosen emerging countries and the major (global) currencies - the euro and the American dollar. The idea of the investigation is taken from a paper by Eun and Lai (2004) describing the pattern of currency co-movement. The shortage of their approach is that it captures only linear dependencies between the analyzed exchange rates. In our investigation, currency linkages are described by using dynamic copula models. This allows us to apply dependence measures which are better suited to quantify dependence in the case of nonelliptical joint conditional distributions and take into account dependencies between extremes.

Practitioners working in financial markets express very often an opinion that currency co-move and the pattern of this co-movement is significantly driven by the competitive influence of the two global currencies, i.e. the US dollar and the euro. This pattern is a crucial factor of the FX market risk because it determines the possibilities of international portfolio diversification. Moreover, the knowledge about the way in which currencies co-move is very important for understanding many macroeconomic phenomena. The investigation of currency market linkages is more complicated than it is in the capital market case. The reason for this is that the analysis of comovement between two currencies requires the consideration of exchange rates which are always calculated against a third currency. The choice of this third currency certainly influences the results of comparison. Despite this difficulty and the fact that the exchange rate fluctuations are subject to different shocks, it is possible to find some pattern of dependencies suggesting that some currencies can co-move in a predictable manner. A special situation occurs when the third currency involved in the analysis is one of the global currencies: the US dollar or the euro. Eun and Lai (2004) confirm the above mentioned opinion that currency co-movement is significantly driven by the competitive influences of major currencies on minor ones. Their analysis is based on the fact that in the case when a minor currency XYZ is driven by the US dollar, the exchange rates XYZ/EUR and USD/EUR co-move very closely (XYZ/EUR means the price of $1 \mathrm{XYZ}$ in euros). In the opposite case i.e. when the XYZ is influenced by the euro, the exchange rates XYZ/USD and EUR/USD show strong interdependence. Eun and Lai (2004) propose to measure the degree to which a currency is exposed to the influence of a major currency, using the so-called "currency beta". Their idea is obviously taken from the notion of the Sharpe beta. The currency beta is a measure of sensitivity of a currency's exchange rate movements to those of a major currency. The disadvantage of their approach is that it only captures the linear part of dependencies. We propose a different description based on very flexible Markov regime switching copula models that allow for a deeper analysis of the currency market linkages. In this framework, the measure of "normal" dependence is dynamic Spearman's rho. Additionally, we obtain information about the pattern of tail dependencies i.e. the dependencies between extremes.

In the paper, we attempt to discover the nature and dynamics of linkages between the Czech koruna (CZK), Indian rupiah (INR), Polish zloty (PLN), Russian ruble (RUB), Turkish lira (TRY), and the global currencies: the US dollar and the euro. The aim of the investigation is to describe the possible changes in the impact of the two major currencies on the considered currencies of the emerging countries during last 16 years. Our results confirm that CZK and PLN are in the euro area of influence. They also suggest that the currencies INR, RUB and TRY seem to be connected with the US dollar. However, the increasing impact of the euro on those currencies is observed. 


\section{Copulas and Dependence Measures}

The usual Pearson linear correlation still plays a significant role in financial econometrics, yet it should be realized that this tool for measuring dependence between financial returns is appropriate only in the case of elliptical distributions (e.g. multivariate normal or multivariate Student's $t$ distributions). Outside this class of distributions a more adequate measure of dependence is provided by the concept of copula. A bivariate copula can be defined as a mapping $C:[0,1] \times[01] \rightarrow[0,1]$ from the unit square into the unit interval which is a distribution function with standard uniform marginal distributions.

Assume that $(X, Y)$ is a 2-dimensional random vector with joint distribution $H$ and marginal distributions $F$ and $G$. Then by a theorem by Sklar (1959) $H$ can be written as

$$
H(x, y)=C(F(x), G(y)) \text {. }
$$

If $F$ and $G$ are continuous, the function $C$ is unique and is given by

$$
C(u, v)=H\left(F^{\leftarrow}(u), G^{\leftarrow}(v)\right),
$$

for $u, v \in[0,1]$, where $F^{\leftarrow}(u)=\inf \{x: F(x) \geq u\}$. In this case, $C$ is called the copula of $H$ or of $(X, Y)$. Since by means of the copula $C$ the marginals and the dependence structure can be separated, it makes sense to interpret the copula as the dependence structure of the vector $(X, Y)$.

The simplest copula is defined by $C^{\Pi}(u, v)=u v$ and it corresponds to independence of marginal distributions. In the empirical part of this paper we will also use the Gaussian, Joe-Clayton, and rotated Joe-Clayton copulas. They are defined as follows:

$$
\begin{aligned}
& C_{\rho}^{\text {Gauss }}(u, v)=\Phi_{\rho}\left(\Phi^{-1}(u), \Phi^{-1}(v)\right), \\
& C_{\kappa, \gamma}^{J-C}(u, v)=1-\left(1-\left(\left[1-(1-u)^{\kappa}\right]^{-\gamma}+\left[1-(1-v)^{\kappa}\right]^{-\gamma}-1\right)^{-1 / \gamma}\right)^{1 / \kappa}, \\
& C_{\kappa, \gamma}^{J-C_{-} r 90}(u, v)=v-C_{\kappa, \gamma}^{\mathrm{J}-\mathrm{C}}(1-u, v) .
\end{aligned}
$$

In formula (3), $\Phi_{\rho}$ denotes the distribution function of standard 2-dimensional normal vector with the linear correlation coefficient $\rho$, and $\Phi$ stands for the standard normal distribution function. The parameters in the Joe-Clayton copula (4) are assumed to satisfy the conditions: $\kappa \geq 1, \gamma>0$. For $\kappa=1$, the Joe-Clayton copula becomes the Clayton copula $C_{\gamma}^{\text {Clayton }}$. In the limit case $\gamma=0$, the Clayton copula approaches the independent copula $C^{\Pi}$ (Nelsen 2006). The rotated version of the Joe-Clayton copula, $C_{\kappa, \gamma}^{J-C_{-} r 90}$, allows for the modeling of negative dependence, which is not possible using the copula $C_{\kappa, \gamma}^{J-C}$.

The density associated to a copula $C$ is a function $c$ defined by

$$
c(u, v)=\frac{\partial^{2} C(u, v)}{\partial u \partial v} \text {. }
$$

In the case of continuous random vector, the copula density $c$ is related to joint density function $h$ by the following canonical representation:

$$
h(x, y)=c(F(x), G(y)) f(x) g(y),
$$

where $F$ and $G$ are the marginal distribution functions, and $f$ and $g$ are the marginal density functions.

If the joint distribution of a random vector $(X, Y)$ is nonelliptical, measures of dependence, which are more appropriate than the linear correlation coefficient, are copula-based ones. An example of such a measure, used in the empirical part of this paper, is Spearman's rho. It can be defined as

$$
\rho_{S}(X, Y)=\rho(F(X), G(Y)),
$$

where $F$ and $G$ are the distribution functions of the variables $X$ and $Y$, respectively (McNeil et al. 2005). If $(X, Y)$ is a vector of continuous random variables with copula $C$, then

$$
\rho_{S}(X, Y)=\rho_{C}=12 \iint_{[0,1]^{2}} C(u, v) d u d v-3
$$

(Nelsen 2006). For the Gaussian copula, $C_{\rho}^{\text {Gauss }}$, Spearman's rho equals $\frac{6}{\pi} \arcsin \frac{1}{2} \rho$. For the Clayton and JoeClayton copulas, it can be computed numerically using (9). Spearman's rhos for the copula $C_{\kappa, \gamma}^{J-C_{-} r 90}$ and $C_{\kappa, \gamma}^{J-C}$ are equal in magnitude and have opposite signs. 
A very important concept relevant to dependence in extreme values is tail dependence (see Joe 1997). If $X$ and $Y$ are random variables with distribution functions $F$ and $G$ then the coefficient of upper tail dependence is defined as follows

$$
\lambda_{U, U}=\lim _{q \rightarrow 1^{-}} P\left(Y>G^{\leftarrow}(q) \mid X>F^{\leftarrow}(q)\right),
$$

provided a limit $\lambda_{U, U} \in[0,1]$ exists. Analogously, the coefficient of lower tail dependence is defined

$$
\lambda_{L, L}=\lim _{q \rightarrow 0^{+}} P\left(Y \leq G^{\leftarrow}(q) \mid X \leq F^{\leftarrow}(q)\right),
$$

provided that a limit $\lambda_{L, L} \in[0,1]$ exists. If $\lambda_{U, U} \in(0,1] \quad\left(\lambda_{L, L} \in(0,1]\right)$, then $X$ and $Y$ are said to exhibit upper (lower) tail dependence. Upper (lower) tail dependence quantifies the likelihood to observe a large (low) value of $Y$ given a large (low) value of $X$. The coefficients of tail dependence depend only on the copula $C$ of $X$ and $Y$ :

$$
\begin{aligned}
& \lambda_{L, L}=\lim _{q \rightarrow 0^{+}} \frac{C(q, q)}{q}, \\
& \lambda_{U, U}=\lim _{q \rightarrow 0^{+}} \frac{\hat{C}(q, q)}{q} .
\end{aligned}
$$

where $\hat{C}(u, v)=u+v-1+C(1-u, 1-v)$. For the Gaussian copula it holds $\lambda_{U, U}=\lambda_{L, L}=0$ (McNeil et al. 2005), meaning asymptotic independence in the tails. In the case of the Joe-Clayton copula, $\lambda_{U, U}=2-2^{1 / \kappa}$ and $\lambda_{L, L}=2^{-1 / \gamma}$ (Patton 2006). Thus upper and lower tail dependence can change freely of each other.

When the components of the random vector $(X, Y)$ display negative dependence it is convenient to consider negative tail dependence. We define the coefficients of negative tail dependence, $\lambda_{U, L}$ and $\lambda_{L, U}$, as follows

$$
\begin{aligned}
& \lambda_{U, L}=\lim _{q \rightarrow 0^{+}} P\left(Y \leq G^{\leftarrow}(q) \mid X>F^{\leftarrow}(1-q)\right), \\
& \lambda_{L, U}=\lim _{q \rightarrow 1^{-}} P\left(Y>G^{\leftarrow}(q) \mid X \leq F^{\leftarrow}(1-q)\right),
\end{aligned}
$$

provided that the limits exist. It can be directly shown that the coefficients of negative tail dependence are invariants of the copula $C$ of the random vector $(X, Y)$ :

$$
\begin{aligned}
& \lambda_{U, L}=\lim _{q \rightarrow 0^{+}} \frac{q-C(1-q, q)}{q}, \\
& \lambda_{L, U}=\lim _{q \rightarrow 0^{+}} \frac{q-C(q, 1-q)}{q} .
\end{aligned}
$$

It follows from (16) and (17) that for the rotated Joe-Clayton copula $C_{\kappa, \gamma}^{\mathrm{J}-\mathrm{r} \_ \text {r90 }}$ the following holds:

$$
\begin{aligned}
& \lambda_{U, L}\left(C_{\kappa, \gamma}^{J-C_{-} r 90}\right)=\lambda_{L, L}\left(C_{\kappa, \gamma}^{J-C}\right)=2^{-1 / \gamma}, \\
& \lambda_{L, U}\left(C_{\kappa, \gamma}^{J-C_{-} r 90}\right)=\lambda_{U, U}\left(C_{\kappa, \gamma}^{J-C}\right)=2-2^{1 / \kappa} .
\end{aligned}
$$

\section{Markov Regime Switching Copula Models}

We model the joint conditional distributions of bivariate returns using copulas, so the evolution of the conditional copula $C_{t}$ has to be specified. In such approach, usually the functional form of the conditional copula is fixed, but its parameters evolve through time (Patton 2004, 2006). In this paper, however, similarly to we assume that there are three regimes in each of which a fixed copula prevails, and they switch according to some Markov chain. Thus the joint conditional distribution of the vector $r_{t}=\left(r_{1, t}, r_{2, t}\right)$ in the applied Markov regime switching copula model (MRSC model) has the following form

$$
r_{t} \mid \Omega_{t-1} \sim C_{S_{t}}\left(F_{1, t}(\cdot), F_{2, t}(\cdot) \mid \Omega_{t-1}\right)
$$

where $S_{t}$ is a homogeneous Markov chain with state space $\{1,2,3\}$. The parameters of the MRSC model are the parameters of univariate models for marginal distributions (ARMA-GARCH), the parameters of copulas $C_{1}$ ,$C_{2}$ and $C_{3}$, and the transition probabilities, $p_{i j}=P\left(S_{t}=j \mid S_{t-1}=i\right),(i, j) \in\{(1,1),(2,2),(3,3),(1,2),(2,1),(3,2)\}$.

The parameters have been estimated by the maximum likelihood method. The main by-product the estimation are the conditional probabilities $P\left(S_{t}=j \mid \Omega_{t-1}\right)$ and $P\left(S_{t}=j \mid \Omega_{t}\right), j=1,2,3$. They are calculated by means of Hamilton's filter (Hamilton, 1994): 


$$
\begin{aligned}
& P\left(S_{t}=j \mid \Omega_{t-1}\right)=\sum_{i=1}^{3} p_{i j} P\left(S_{t-1}=i \mid \Omega_{t-1}\right), \\
& P\left(S_{t}=j \mid \Omega_{t}\right)=\frac{c_{j}\left(u_{t} \mid S_{t}=j, \Omega_{t-1}\right) P\left(S_{t}=j \mid \Omega_{t-1}\right)}{\sum_{i=1}^{3} c_{i}\left(u_{t} \mid S_{t}=i, \Omega_{t-1}\right) P\left(S_{t}=i \mid \Omega_{t-1}\right)}
\end{aligned}
$$

where $p_{13}=1-p_{11}-p_{12}, p_{23}=1-p_{21}-p_{22}, p_{31}=1-p_{32}-p_{33}, u_{t}=\left(u_{1, t}, u_{2, t}\right)^{\prime}$,

$$
u_{1, t}=F_{1, t}\left(r_{1, t}\right), \quad u_{2, t}=F_{2, t}\left(r_{2, t}\right) \text {, }
$$

and $c_{j}\left(\cdot \mid S_{t}=j, \Omega_{t-1}\right)$ is the density of the conditional copula coupling the conditional marginal distributions in regime $j, j=1,2,3$. The maximized log-likelihood function has of the form

$$
\begin{aligned}
& L=\sum_{t=1}^{T} \ln \left(\sum_{j=1}^{3} c_{j}\left(u_{t} \mid S_{t}=j, \Omega_{t-1} ; \widetilde{\theta}_{j}\right) P\left(S_{t}=j \mid \Omega_{t-1} ; \widetilde{\theta}_{j}\right)\right) \\
& +\sum_{t=1}^{T} \ln \left(f_{1, t}\left(r_{1, t} \mid \Omega_{t-1} ; \theta_{1}\right)\right)+\sum_{t=1}^{T} \ln \left(f_{2, t}\left(r_{2, t} \mid \Omega_{t-1} ; \theta_{2}\right)\right)
\end{aligned}
$$

where $f_{1, t}$ and $f_{2, t}$ are the density functions corresponding to $F_{1, t}$ and $F_{2, t}$, fitted using the AR-GARCH models.

Presenting our empirical results, we use the so-called smoothed probabilities which can be obtained from the predicted and filtered probabilities by using the backward recursion:

$$
P\left(S_{t}=j \mid \Omega_{T}\right)=P\left(S_{t}=j \mid \Omega_{t}\right) \sum_{i=1}^{3} \frac{p_{j i} P\left(S_{t+1}=i \mid \Omega_{T}\right)}{P\left(S_{t+1}=i \mid \Omega_{t}\right)}, \quad t=T-1, \ldots, 1,
$$

where $T$ denotes the length of the sample.

The models are estimated by the two-step maximum likelihood method. First we fit univariate ARMAGARCH models to the return data. The obtained standardized residuals are transformed to uniform variates, using the theoretical cumulative distribution functions. After that copula models are fitted and estimates of dynamic Spearman's rho and the lower and upper tail coefficients and the coefficients of negative tail dependence are calculated using the smoothed probabilities.

\section{The Data, Analysis, and Results}

As it was mentioned before, our investigations follows the idea proposed by Eun and Lai (2004). This idea is that in the case when a minor currency XYZ is driven by the US dollar, the exchange rates XYZ/EUR and USD/EUR co-move very closely. In the opposite case, i.e. when the XYZ is influenced by the euro, the exchange rates XYZ/USD and EUR/USD show strong interdependence. Applying very flexible 3-regime Markov switching copula models, allows us to describe different types of dependence and capture temporal changes in the impact of the global currencies on the analyzed minor ones.

The analysis is based on the percentage daily logarithmic returns of the exchange rates of chosen currencies against the USD and euro. The considered currencies are CZK, TRY, RUB, PLN, INR, and the period under scrutiny is from January 3, 1997 to May 24, 2013 (about 4200 observations). For the period before 1999, exchange rates against the ecu are applied. The exception is the Russian ruble, for which the sample begins from January 3, 2002.

The steps of the presented analysis were as follows. First, to each of the investigated return series we fitted a univariate ARMA-GARCH model. Because the data under scrutiny come from a long period, the fitted models are usually the asymmetric GARCH (EGARCH, APARCH or GJR-GARCH) ones with a Student's $t$ or skewed Student's $t$ distribution for the innovations (Lambert and Laurent 2001). The obtained standardized residuals were routinely examined for the presence of desirable distributional and serial properties. Next, the standardized residuals from the ARMA-GARCH models were transformed by means of the corresponding distribution functions into the series of the form (23), and the MRSC models were fitted by the maximum likelihood method, using the first summand in (24). We tried to apply copulas from various families, and our final choice is the effect of taking into account the obtained values of the information criteria, and the results of the performed likelihood ratio tests where it was applicable. All the calculations were done using G@RCH 6.0 package (Laurent 2009) and the Matlab software. Due to space limit, we do not present here the estimates of univariate models, and focus only on the results concerning estimation of the MRSC models. 
In Tables 1-5 we present the parameter estimates for the fitted MRSC models. The plots in Figures 1-10 show the dynamics of the applied dependence measures. A general observation is that CZK, PLN are in the euro area of influence, whereas INR, RUB and TRY seem to be connected with the USD dollar.

The linkages of the Czech koruna with the euro, measured by means of Spearman's rho, oscillate from 0.6 to 0.9 (Figure 1), what indicates the stable level of dependence. They are described by 2-regime copula model with the Gaussian copula in each regime, what indicates no tail dependence. Dependence between the CZK/EUR and USD/EUR is very weak or even, sometimes, negative (Figures 2, 3).

\begin{tabular}{|c|c|c|c|c|c|c|}
\hline $\begin{array}{l}\text { CZK/USD } \\
\text { EUR/USD }\end{array}$ & Regime 1 & Regime 2 & $\begin{array}{l}\text { CZK/EUR } \\
\text { USD/EUR }\end{array}$ & Regime 1 & Regime 2 & Regime 3 \\
\hline Copula & $C_{\rho}^{\text {Gauss }}$ & $C_{\rho}^{\text {Gauss }}$ & Copula & $C_{\rho}^{\text {Gauss }}$ & $C^{P I}$ & $C_{\kappa, \gamma}^{\text {Joe-Clayto } \theta 0}$ \\
\hline$\rho$ & $\begin{array}{l}0.5927 \\
(0.0197)\end{array}$ & $\begin{array}{l}0.8879 \\
(0.0052)\end{array}$ & $\rho$ & $\begin{array}{l}0.2784 \\
(0.0227)\end{array}$ & & \\
\hline$\kappa$ & & & $\kappa$ & & & $\begin{array}{l}4.0461 \\
(0.0174) \\
\end{array}$ \\
\hline$\gamma$ & & & $\gamma$ & & & $\begin{array}{l}4.8977 \\
(0.0085)\end{array}$ \\
\hline & \multicolumn{2}{|c|}{ Transition probabilities } & & \multicolumn{3}{|c|}{ Transition probabilities } \\
\hline Regime 1 & 0.9689 & 0.0311 & Regime 1 & 0.9989 & 0.0011 & 0.0000 \\
\hline Regime 2 & & 0.9831 & Regime 2 & 0.0001 & 0.9956 & 0.0044 \\
\hline & & & Regime 3 & 0.0051 & 0.0494 & 0.9455 \\
\hline
\end{tabular}

Table 1. CZK. Parameter estimates for MRSC models.

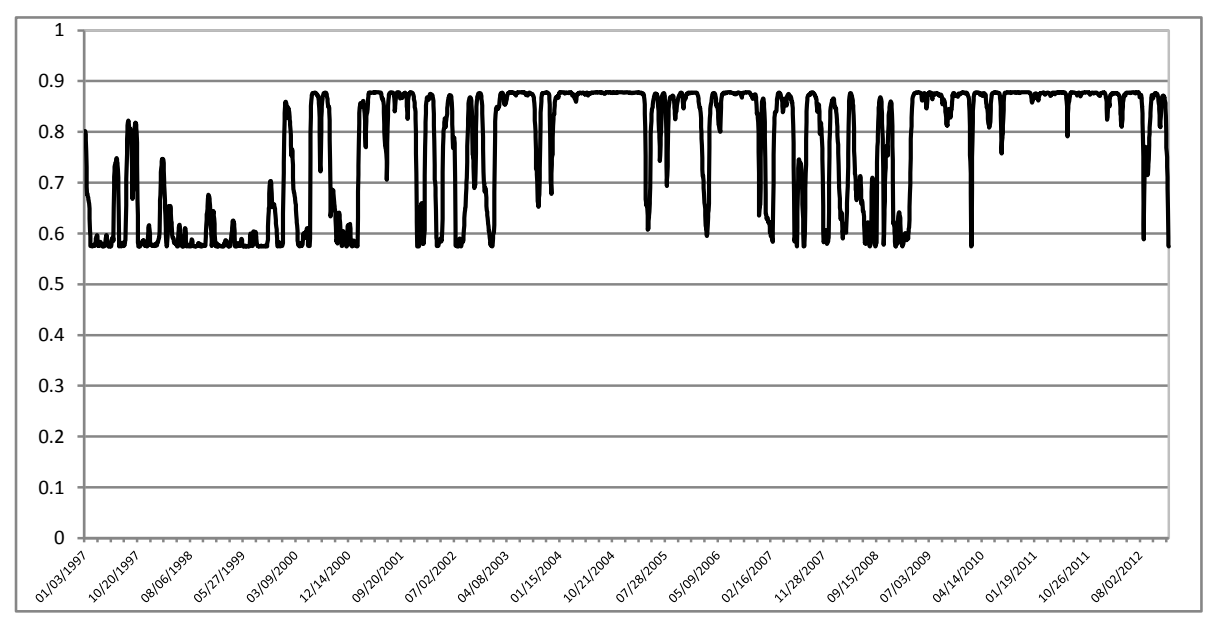

Figure 1. Linkages between CZK and EUR. Spearman's rho for (CZK/USD, EUR/USD)

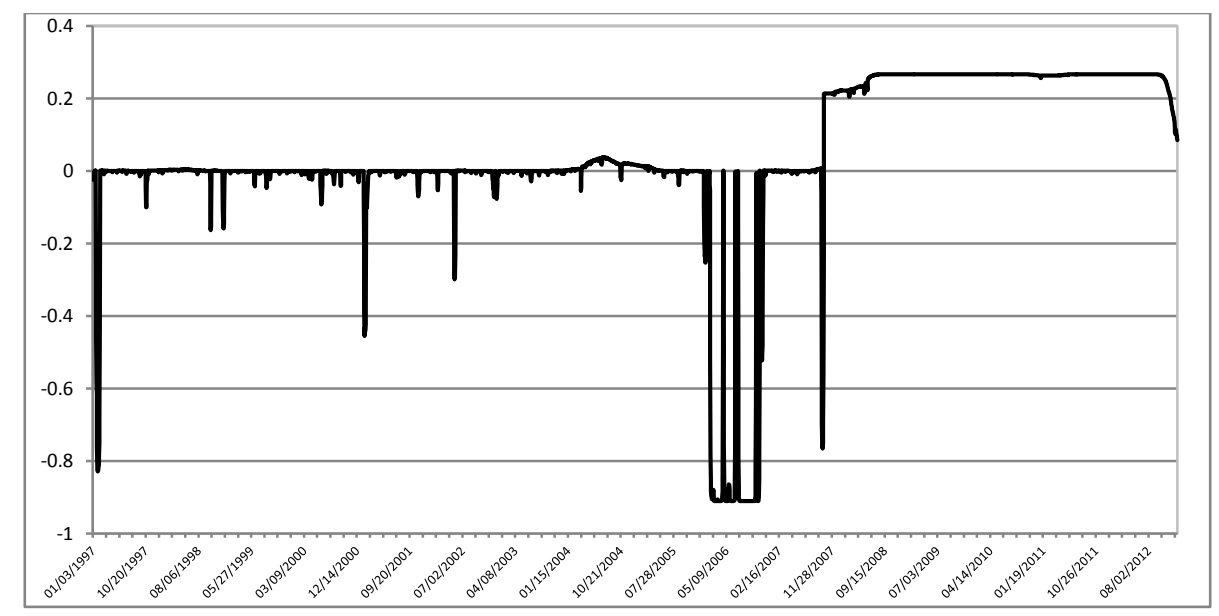

Figure 2. Linkages between CZK and the USD. Spearman's rho for (CZK/EUR, USD/EUR) 


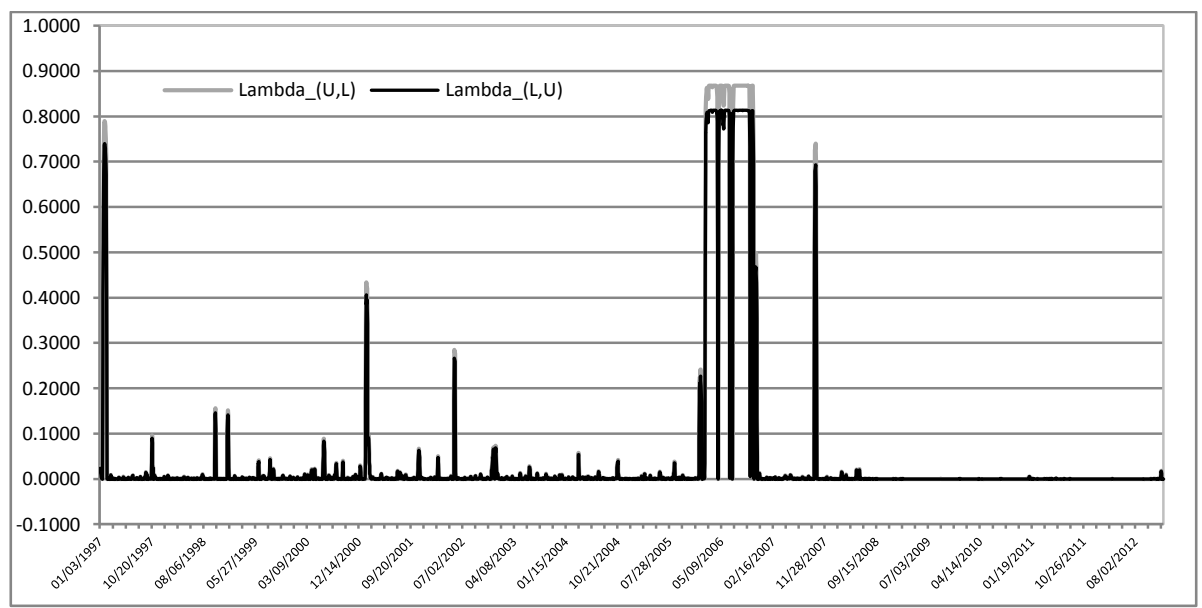

Figure 3. Linkages between CZK and USD. Dependence in tails for (CZK/EUR, USD/EUR)

The dependence between the Indian rupiah and the euro is described by means of 2-regime MRSC model. In regime 1, the Gaussian copula with the correlation coefficient 0.2274 is applied, and in regime 2, the independence copula fits. This means that the dependence between INR and EUR is very weak. Figure 6 shows, however, that the level of dependence jumped in 2004 from negative values to 0.2 . The linkages with the USD are stable with strong dependence in tails (Table 2, Figure 4).

\begin{tabular}{|c|c|c|c|c|c|c|}
\hline $\begin{array}{l}\text { INR/USD } \\
\text { EUR/USD }\end{array}$ & Regime 1 & Regime 2 & $\begin{array}{l}\text { INR/EUR } \\
\text { USD/EUR }\end{array}$ & Regime 1 & Regime 2 & Regime 3 \\
\hline Copula & $C_{\rho}^{\text {Gauss }}$ & $C^{P I}$ & Copula & $C_{\rho}^{\text {Gauss }}$ & $C_{\gamma}^{\text {Clayton }}$ & $C_{k, y}^{\text {Joe-Clayon }}$ \\
\hline$\rho$ & $\begin{array}{l}0.2274 \\
(0.0180)\end{array}$ & & $\rho$ & $\begin{array}{l}0.8538 \\
(0.0160)\end{array}$ & $\begin{array}{l}0.2836 \\
(0.1566) \\
\end{array}$ & \\
\hline$\kappa$ & & & $\kappa$ & & & $\begin{array}{l}4.5441 \\
(0.0001)\end{array}$ \\
\hline$\gamma$ & & & $\gamma$ & & & $\begin{array}{l}5.0000 \\
(0.0003)\end{array}$ \\
\hline & \multicolumn{2}{|c|}{ Transition probabilities } & & \multicolumn{3}{|c|}{ Transition probabilities } \\
\hline Regime 1 & 0.9997 & 0.0003 & Regime 1 & 0.9527 & 0.0337 & 0.0036 \\
\hline Regime 2 & 0.0003 & 0.9997 & Regime 2 & 0.0203 & 0.9797 & 0.0000 \\
\hline & & & Regime 3 & 0.0000 & 0.0045 & 0.9955 \\
\hline
\end{tabular}

Table 2. INR. Parameter estimates for MSC models.

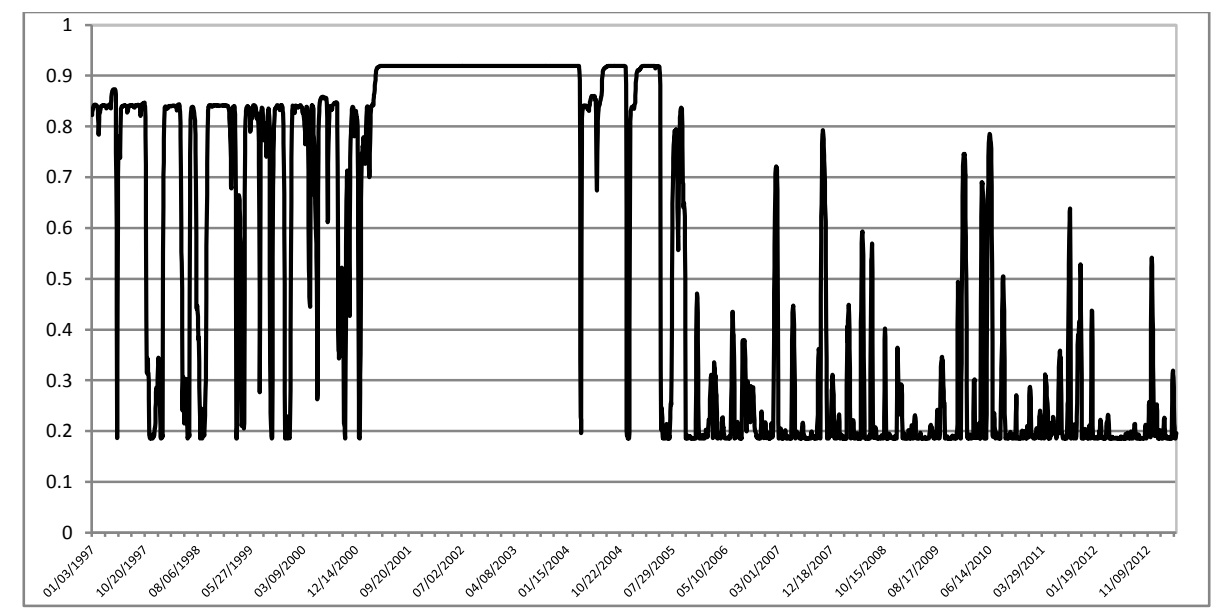

Figure 4. Linkages between INR and USD. Spearman's rho for (INR/EUR, USD/EUR) 


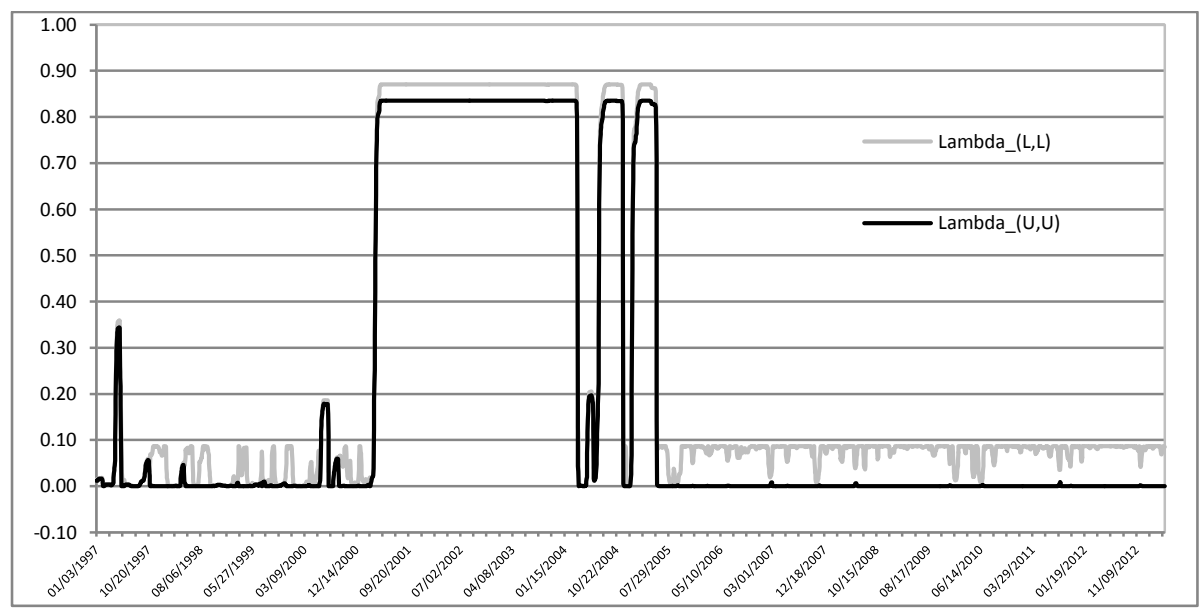

Figure 5. Linkages between INR and the USD. Dependence in tails for (INR /EUR, USD/EUR)

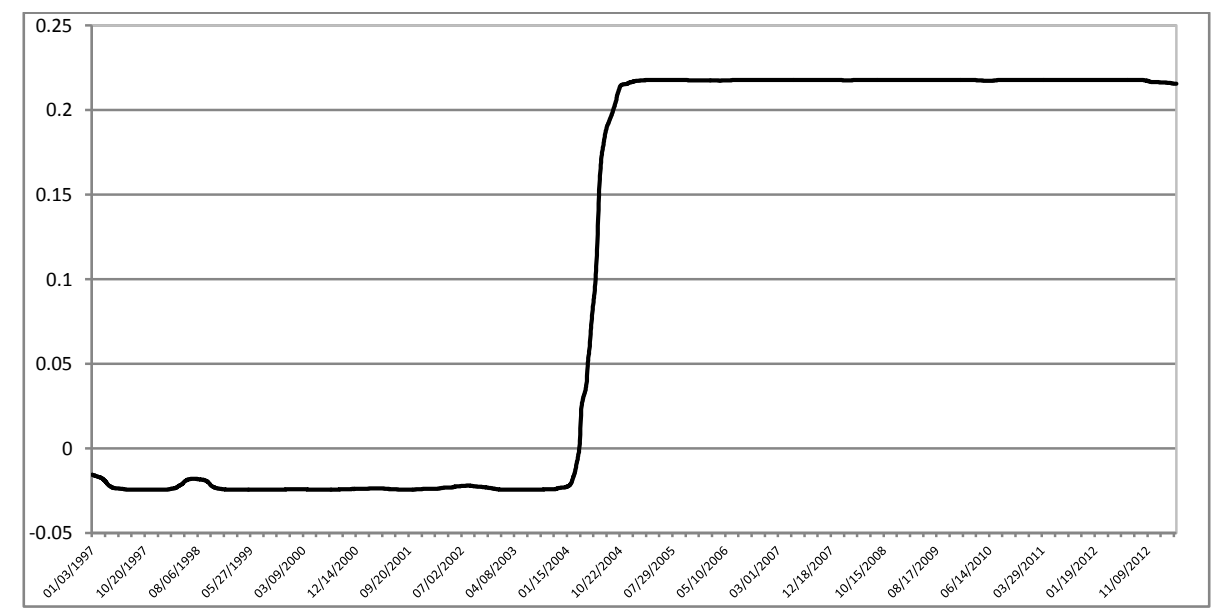

Figure 6. Linkages between INR and EUR. Spearman's rho for (INR/USD, EUR/USD)

In the case of the Polish zloty, we observe a spectacular decrease in the linkages (from 0.4 to negative values, even -0.4) with US dollar after Poland's EU joining in 2004. The connections with the euro are described by means 3- regime MRSC model with the Gaussian copula in regimes 1 and 2, and the Joe-Clayton copula in regime 3 . In the case of the dependence with the US dollar, the applied copulas are the independence, the JoeClayton, and the rotated Joe-Clayton.

\begin{tabular}{|c|c|c|c|c|c|c|c|}
\hline $\begin{array}{l}\text { PLN/USD } \\
\text { EUR/USD }\end{array}$ & Regime 1 & Regime 2 & Regime 3 & $\begin{array}{l}\text { PLN/EUR } \\
\text { USD/EUR }\end{array}$ & Regime 1 & Regime 2 & Regime 3 \\
\hline Copula & $C_{\rho}^{\text {Gauss }}$ & $C_{\rho}^{\text {Gauss }}$ & $C_{\kappa, \gamma}^{\text {Joe-Clayton }}$ & Copula & $C^{P I}$ & $C_{k, \gamma}^{\text {Joe-Clayon }}$ & $C_{\kappa \gamma}^{\text {Joe-Clayton } 90}$ \\
\hline$\rho$ & $\begin{array}{l}0.3409 \\
(0.0219)\end{array}$ & $\begin{array}{l}0.7746 \\
(0.0106)\end{array}$ & & $\rho$ & & & \\
\hline$\kappa$ & & & $\begin{array}{l}1.1751 \\
(0.0708)\end{array}$ & $\kappa$ & & $\begin{array}{l}1.2738 \\
(0.0367)\end{array}$ & $\begin{array}{l}1.1622 \\
(0.0422)\end{array}$ \\
\hline$\gamma$ & & & $\begin{array}{l}0.1693 \\
(0.0893)\end{array}$ & $\gamma$ & & $\begin{array}{l}0.4942 \\
(0.0408)\end{array}$ & $\begin{array}{l}0.5764 \\
(0.0656) \\
\end{array}$ \\
\hline & \multicolumn{3}{|c|}{ Transition probabilities } & & \multicolumn{3}{|c|}{ Transition probabilities } \\
\hline Regime 1 & 0.9990 & 0.0000 & 0.0010 & Regime 1 & 0.9859 & 0.0005 & 0.0136 \\
\hline Regime 2 & 0.0000 & 0.9520 & 0.0480 & Regime 2 & 0.0000 & 0.9997 & 0.0003 \\
\hline Regime 3 & 0.0018 & 0.2053 & 0.7929 & Regime 3 & 0.0127 & 0.0001 & 0.9873 \\
\hline
\end{tabular}

Table 3. PLN. Parameter estimates for MRSC models. 


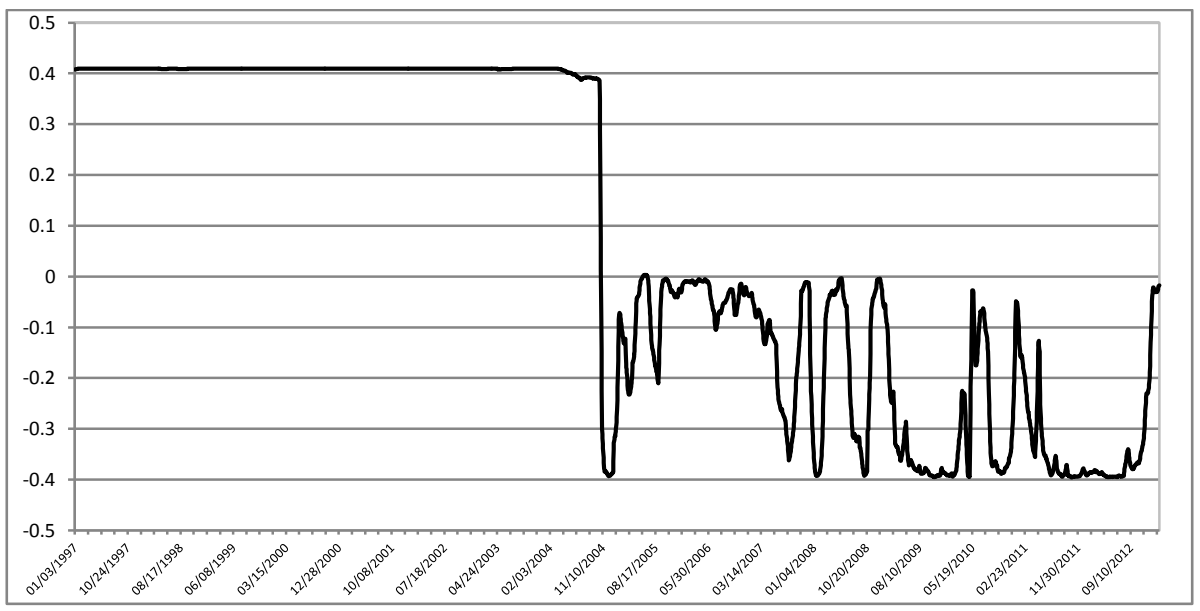

Figure 7. Linkages between PLN and USD. Spearman's rho for (PLN/EUR, USD/EUR)

In the case of the Russian ruble, we analyze shorter time series - from 2002 to 2013. Our results show that during this period the RUB is switching between the areas of influence of the major currencies (Figures 8,9). In the case of linkages with the euro, tail dependence appears (the Joe-Clayton copula in regime 3 ). There is no dependence in tails with the USD against the euro.

\begin{tabular}{|c|c|c|c|c|c|c|c|}
\hline $\begin{array}{l}\text { RUB/USD } \\
\text { EUR/USD }\end{array}$ & Regime 1 & Regime 2 & Regime 3 & $\begin{array}{l}\text { RUB/EUR } \\
\text { USD/EUR }\end{array}$ & Regime 1 & Regime 2 & Regime 3 \\
\hline Copula & $C^{P I}$ & $C_{\rho}^{\text {Gauss }}$ & $C_{\kappa,}^{\text {Joe-Clyyon }}$ & Copula & $C_{\rho}^{\text {Gauss }}$ & $C_{\rho}^{\text {Gauss }}$ & $C^{P I}$ \\
\hline$\rho$ & & $\begin{array}{l}0.5524 \\
(0.0177)\end{array}$ & & $\rho$ & $\begin{array}{l}0.6432 \\
(0.0179)\end{array}$ & $\begin{array}{l}0.9013 \\
(0.0103)\end{array}$ & \\
\hline$\kappa$ & & & $\begin{array}{l}2.0319 \\
(0.1395)\end{array}$ & $\kappa$ & & & \\
\hline$\gamma$ & & & $\begin{array}{l}1.4248 \\
(0.1687)\end{array}$ & $\gamma$ & & & \\
\hline & \multicolumn{3}{|c|}{ Transition probabilities } & & \multicolumn{3}{|c|}{ Transition probabilities } \\
\hline Regime 1 & 0.9977 & 0.0023 & 0.0000 & Regime 1 & 0.9867 & 0.0000 & 0.0133 \\
\hline Regime 2 & 0.0014 & 0.9979 & 0.0007 & Regime 2 & 0.0000 & 0.9891 & 0.0109 \\
\hline Regime 3 & 0.0000 & 0.0026 & 0.009973 & Regime 3 & 0.0372 & 0.0042 & 0.9585 \\
\hline
\end{tabular}

Table 4. RUB. Parameter estimates for MRSC models.

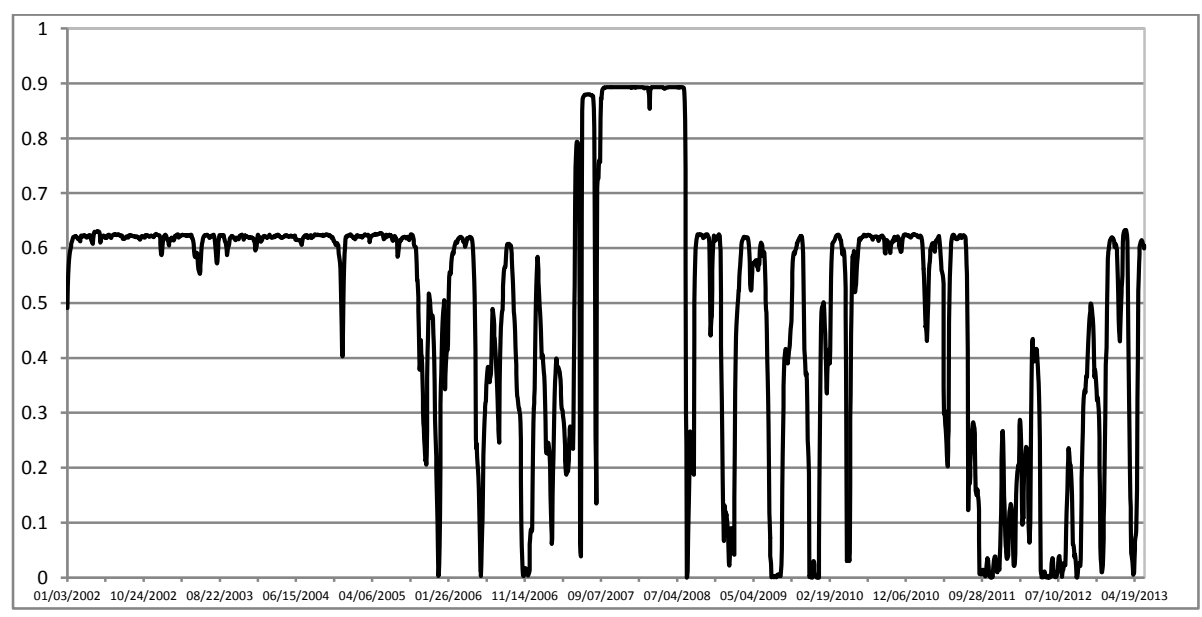

Figure 8. Linkages between RUB and USD. Spearman's rho for (RUB/EUR, USD/EUR) 


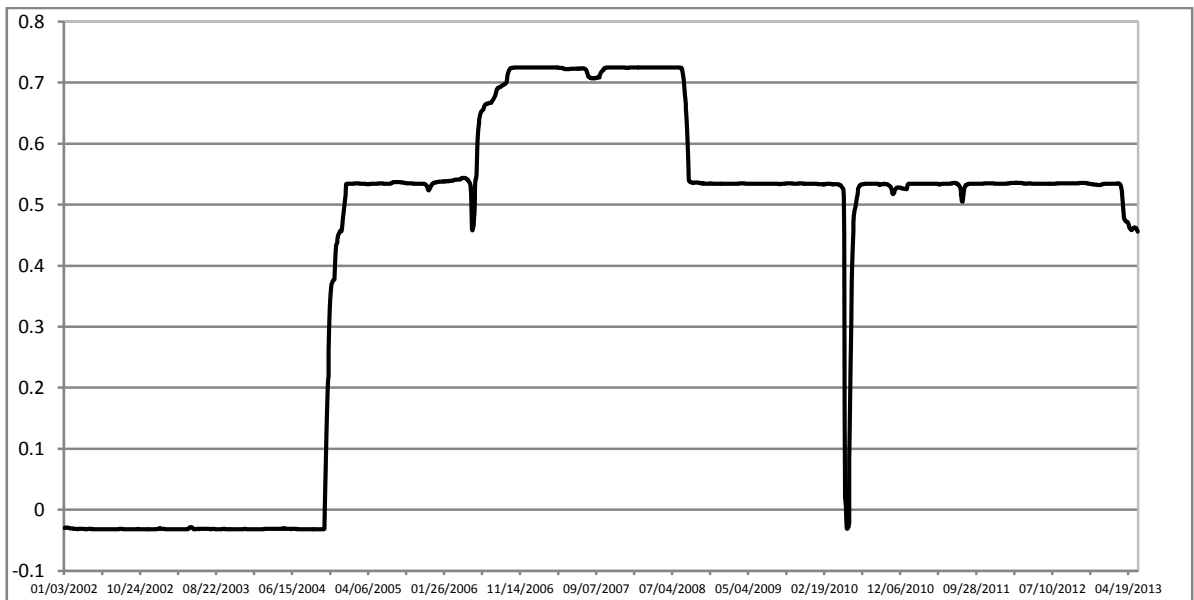

Figure 9. Linkages between the RUB and the EUR. Spearman's rho for (RUB/USD, EUR/USD)

The linkages between the Turkish lira and US dollar, against the euro, oscillate from negative values to even 0.75, exhibiting very strong dynamics (Figure 10). From 2001, the periods with Spearman's rho lower than 0.45 prevail. The fitted MRSC model is 3-regime with the Gaussian, Joe-Clayton and rotated Joe-Clayton copulas (Table 5). A rather surprising result is that the dependence between the TRY and EUR, against the USD, are described by a static Joe-Clayton copula model, showing stable, non sensitive on market situation pattern of dependence. The value of Spearman's rho is 0.3172 and tail dependence coefficients are 0.0915 for lower tail and 0.2490 for upper one. This means that extreme increases of the EUR/USD impacts stronger on the TRY/USD than extreme decreases.

\begin{tabular}{|c|c|c|c|c|c|}
\hline $\begin{array}{l}\text { TRY/USD } \\
\text { EUR/USD }\end{array}$ & Regime 1 & $\begin{array}{l}\text { TRY/EUR } \\
\text { USD/EUR }\end{array}$ & Regime 1 & Regime 2 & Regime 3 \\
\hline Copula & $C_{k y}^{\text {Joe-Clayon }}$ & Copula & $C_{\rho}^{\text {Gauss }}$ & $C_{k, y}^{\text {Joe Clayon }}$ & $C_{\kappa y}^{\text {Joee-Cayton } 90}$ \\
\hline$\rho$ & & $\rho$ & $\begin{array}{l}0.4891 \\
(0.0381)\end{array}$ & & \\
\hline$\kappa$ & 1.2373 & $\kappa$ & & $\begin{array}{l}2.3201 \\
(0.0807)\end{array}$ & $\begin{array}{l}1.0123 \\
(0.0205)\end{array}$ \\
\hline$\gamma$ & 0.2898 & $\gamma$ & & $\begin{array}{l}1.2178 \\
(0.0643)\end{array}$ & $\begin{array}{l}0.0939 \\
(0.0467)\end{array}$ \\
\hline & & Regime 1 & 0.9524 & 0.0054 & 0.0422 \\
\hline & & Regime 2 & 0.0064 & 0.9935 & 0.0000 \\
\hline & & Regime 3 & 0.1203 & 0.0000 & 0.8796 \\
\hline
\end{tabular}

Table 5. TRY. Parameter estimates for MRSC models.

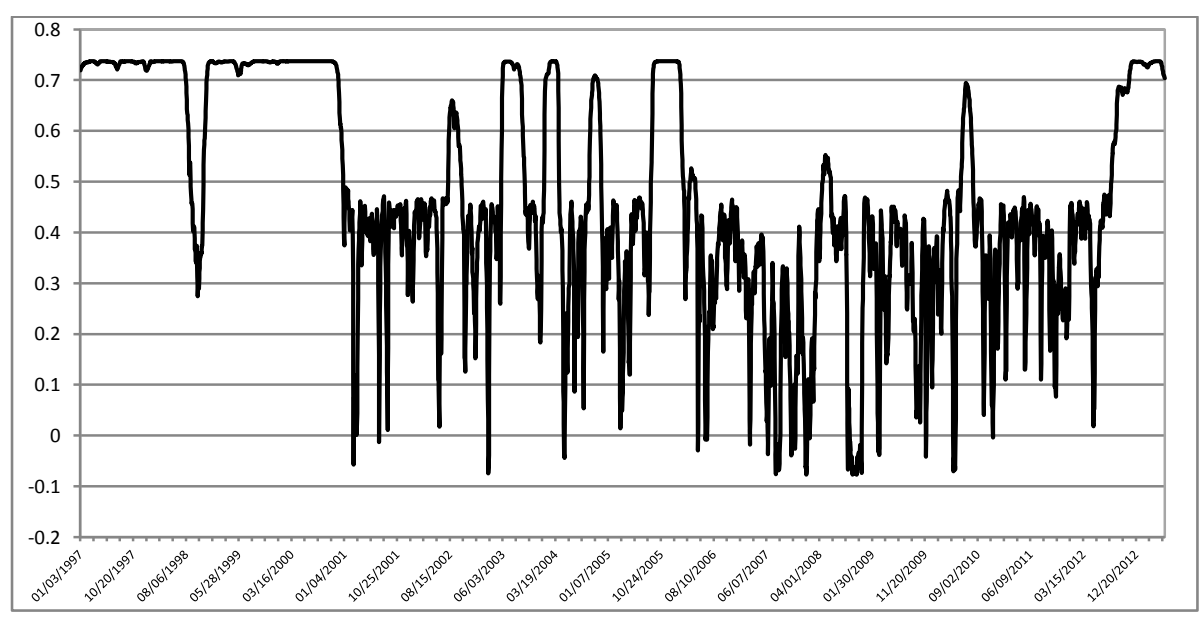

Figure 10. Linkages between the TRY and the USD. Spearman's rho for (TRY/EUR,USD/EUR) 


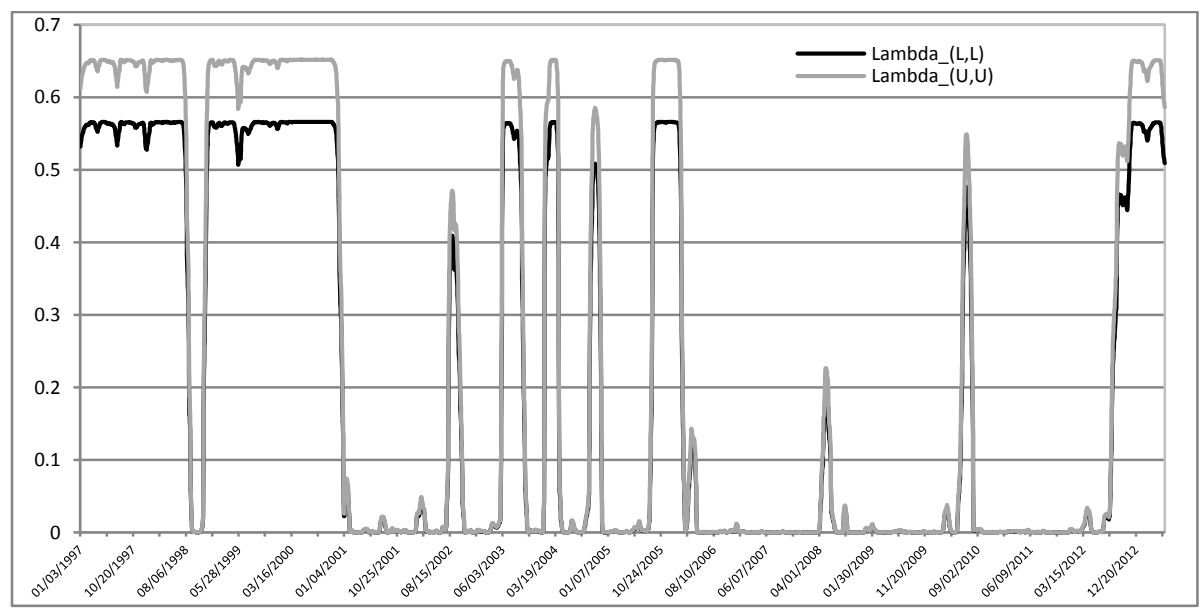

Figure 11. Linkages between the TRY and the USD. Dependence in tails for (TRY /EUR,USD/EUR)

\section{Conclusions}

The aim of the analysis presented in this paper was to describe the pattern of dynamic linkages between the chosen currencies (the Czech koruna, Indian rupiah, Polish zloty, Russian ruble and Turkish lira) and two global currencies - the American dollar and the euro. The applied approach follows the idea by Eun and Lai (2004) that in the case when a minor currency XYZ is driven by the US dollar, the exchange rates XYZ/EUR and USD/EUR co-move very closely. In the opposite case, i.e. when the $\mathrm{XYZ}$ is influenced by the euro, the exchange rates $\mathrm{XYZ/USD}$ and EUR/USD show strong interdependence. To describe the strength and dynamics of dependence between the returns of the analyzed exchange rates we apply very flexible 3-regime Markov switching copula models. The considered dependence measures are dynamic Spearman's rho and dynamic coefficients of dependence tails. A general observation is that the impact of the US dollar and the euro is changing due to globalization process and is sensitive on the market situation. Our results confirm that CZK and PLN are in the euro area of influence. They also indicate that INR, RUB and TRY seem to be connected with the US dollar, even though the increasing impact of the euro on these currencies is observed.

\section{References}

- $\quad$ Eun, C.S., Lai S.-C. S. (2004), “The Currency Co-movement”, Georgia Institute of Technology Working Paper 21.

- Garcia, R., Tsafack, G. (2006), "Dependence Structure and Extreme Comovements in International Equity and Bond Markets", Journal of Banking \& Finance, Vol. 35, pp. 1954-1970.

- Hamilton, J.D. (1994), Time Series Analysis, Princeton University Press, Princeton.

- Joe, H. (1997), Multivariate Models and Dependence Concepts, Chapman and Hall, London.

- $\quad$ Lambert, P., Laurent, S. (2001), "Modelling financial time series using GARCH-type models with a skewed Student distribution for the innovations", Institut de Statistique, Université Catholique de Louvain, Discussion Paper 0125.

- Laurent, S. (2009), Estimating and Forecasting ARCH Models Using G@RCH 6, Timberlake Consultants, London.

- McNeil, A.J., Frey, A., Embrechts, P. (2005), Quantitative Risk Management, Princeton University Press, Princeton.

- Nelsen, R.B. (2006), An Introduction to Copulas, Springer, New York.

- $\quad$ Patton, A.J. (2004), “On the Out-of-Sample Importance of Skewness and Asymmetric Dependence for Asset Allocation”, Journal of Financial Econometrics, Vol. 2, pp.130-168.

- Patton, A.J. (2006), “Modelling Asymmetric Exchange Rate Dependence”, International Economic Review, Vol. 47, pp. 527-556.

- Sklar, A. (1959), "Fonctions de répartition à n dimensions et leurs marges", Publications de l'Institut Statistique de l'Université de Paris, Vol. 8, pp. 229-231. 